\title{
Quantization via Real Polarization of the Moduli Space of Flat Connections and Chern-Simons Gauge Theory in Genus One
}

\author{
Jonathan Weitsman $\star$ \\ Department of Mathematics, M.I.T., Cambridge, MA 02139, USA and \\ Department of Physics, Harvard University, Cambridge, MA 02138, USA
}

Received June 6, 1990; in revised form September 24, 1990

\begin{abstract}
We study the quantization of the moduli space of flat connections on a surface of genus one, using the real polarization of this space described in [10]. The quantum wave functions in this formalism are exponential functions supported along the integral fibres of the polarization. The space of wave functions obtained in this way is isomorphic to a space of theta functions. We use our construction to construct part of what may be a topological field theory in genus one, and to compute the associated invariants of some three manifolds. These computations agree with those of Witten [12], but the invariants are expressed as sums of quantities computed at a discrete set of connections with curvature concentrated on a link in the three manifold. A similar prescription is used to produce knot invariants.
\end{abstract}

\section{Introduction}

In [10] we showed that the moduli space $\bar{S}_{g}$ of flat $S U(2)$ connections on a twomanifold $\Sigma^{g}$ of genus $g$ possessed, in addition to the standard Kähler polarizations, a real polarization. One motivation for this study was that the quantization of this system in this real polarization may give some new insight into the structure of the conformal field theory related to this system, and may be a useful method to study Witten's quantum field theoretic interpretation of the Jones polynomial, with which it is intimately connected.

In this paper, we continue this program by studying the quantization of this system, where $S U(2)$ is replaced by any compact Lie group $G$, on a two-manifold of genus one. In this case the quantization may be carried out explicitly, and indeed is almost trivial. Nonetheless it exhibits many of the expected properties of topological quantum field theory, as axiomatized by Atiyah, and gives a number of other results. These are briefly as follows.

* Supported by NSF Mathematical Sciences Postdoctoral Research Fellowship DMS 88-07291 
First, the quantum Hilbert space $\mathscr{H}_{G}^{k}$, where $k \in \mathbb{Z}$ is the power of the relevant prequantum line bundle, and $G$ is a compact lie group, may be taken to consist of sections of the line bundle supported on certain Lagrangian subvarieties of the moduli space. This space is seen to have a natural linear isomorphism with the space $\mathscr{H}_{\mathbf{G} \text {; hol }}^{k}$ obtained by quantization of this system in a holomorphic polarization. Of course, the space $\mathscr{H}_{G \text {; hol }}^{k}$ depends on a modular parameter $\tau$, which specifies the Kähler polarization of $\bar{S}_{1}$ used to obtain it; and so, the linear isomorphism with $\mathscr{H}_{G}^{k}$ is also $\tau$-dependent. This isomorphism with a fixed linear space exhibits the independence of the space $\mathscr{H}_{G \text {; hol }}^{k}$ of the choice of polarization $[3,9]$ in a natural way. The quantization in a holomorphic polarization may be shown [4] to yield a Hilbert space isomorphic to the space of Weyl-Kac characters for the affine Lie algebra associated to $G$; in a real polarization these are replaced by certain exponential functions supported on Lagrangian subvarieties of the moduli space. They exhibit the well known Kac shifts for the weights of representations of the affine lie algebras [6].

Second, the modular group $S L(2, \mathbb{Z})$ acts on $\mathscr{H}_{G}^{k}$ (as it does on $\mathscr{H}_{G ; \text { hol }}^{k}$ ), and in fact $\mathscr{H}_{G}^{k}$ gives a natural projective representation of $S L(2, \mathbb{Z})$-isomorphic of course to the representation given by the Weyl-Kac characters referred to above. However in our formulation the action of the modular group has a simple geometrical interpretation. The standard generators $S, T$ of $S L(2, \mathbb{Z})$ have different behaviors on the real polarization of [10]. The generator $T=\left(\begin{array}{ll}1 & 1 \\ 0 & 1\end{array}\right)$ preserves the polarization, and acts on $\mathscr{H}_{G}^{k}$ as a diagonal matrix; while the generator $S=\left(\begin{array}{cc}0 & 1 \\ -1 & 0\end{array}\right)$ does not preserve the polarization, and its action must be constructed using the BKS pairing. This yields à priori only a projective representation of $S L(2, \mathbb{Z})$, which we verify is in fact a true representation.

Finally, we come to topological quantum field theory. We construct part of what we expect to be a topological quantum field theory by associating to a twomanifold of genus one the space $\mathscr{H}_{G}^{k}$ described above. In view of the isomorphism described above between the quantizations obtained from the real and holomorphic polarization, this choice is equivalent to that of [12]. However, the close association between elements of our quantization and Lagrangian subvarieties of the moduli space enables us to take a step towards constructing another important constituent of topological field theory, which is the assignment of an element $V(Y) \in \mathscr{H}_{G}^{k}$ to a three-manifold $Y$ with boundary $\partial Y=\Sigma$. This is obtained roughly as follows. Any presentation of the surface $\Sigma^{1}$ as the boundary of a three-manifold $Y^{3}$ obtained by a Heegaard splitting of a three-manifold gives rise to a natural Lagrangian subvariety $L_{Y}$ of $\bar{S}_{1}$. The space $L_{Y}$ is related to the Lagrangian subvariety $L_{S^{1} \times D^{2}}$, corresponding to the standard solid torus, by the action of an element of the modular group $S L(2, \mathbb{Z})$. On the other hand, we shall see that corresponding to the presentation $\Sigma^{1}=\partial\left(S^{1} \times D^{2}\right)$ there corresponds a natural element $V\left(S^{1} \times D^{2}\right) \in \mathscr{H}_{G}^{k}$. Hence, we may use the action of $S L(2, \mathbb{Z})$ described above to obtain an element of $\mathscr{H}_{G}^{k}$ corresponding to $Y$. In fact, we can use the action of $S L(2, \mathbb{Z})$ to obtain such an element (up to a multiplicative constant) for any $Y^{3}$ with $\partial Y^{3}=\Sigma^{1}$, so long as the (isotropic) subvariety $L_{Y} \subset \bar{S}_{1}$ is Lagrangian.

Of course, to check that these results actually correspond to a topological quantum field theory would require the construction to be generalized to higher genus. This we are not able to do. We do however have the following suggestive 
application. Let $M=Y_{+}^{3} \cup_{\Sigma^{1}} Y_{-}^{3}$ be a Heegaard splitting of a three manifold $M$ along $\Sigma^{1}$. The axioms of topological field theory [1] show that the number

$$
Z(M)=\left\langle V\left(Y_{+}\right), V\left(Y_{-}\right)\right\rangle
$$

obtained from a topological quantum field theory is an invariant of the manifold $M$. We use our explicit formula for $V(Y)$ to compute the putative invariant for

$$
M=S^{3}=S^{1} \times D^{2} \cup_{S^{1} \times S^{1}} S^{1} \times D^{2} .
$$

We reproduce Witten's result [12] for the three sphere; but as $V\left(M_{+}\right), V\left(M_{-}\right)$are, as sections of a line bundle over $\bar{S}_{1}$, supported on transverse Lagrangian subvarieties, we see that this invariant is simply the sum of torsions computed at a finite number [in the case of $G=S U(2)$, two] points of $\bar{S}_{1}$.

To apply our results to produce invariants of knots in $S^{3}$, we note that our methods allow us to assign an element of $\mathscr{H}_{G}^{k}$ not only to a three manifold $Y$ bounding $\Sigma^{1}$, but also in some special cases to a pair $(Y, L)$ consisting of a bounding three manifold $Y$ and an embedded circle $L \subset Y$, labeled by a representation of the group $G$. We obtain an invariant of a knot in $S^{3}$ by producing a splitting of $S^{3}$ along the knot torus, thus obtaining two elements of $\mathscr{H}_{G}^{k}$ : one corresponding to the "interior" pair $\left(S^{1} \times D^{2}, K\right)$, and the other corresponding to its complement $Y$, which in this case is guaranteed to correspond to a Lagrangian subvariety $L_{Y} \subset \bar{S}_{1}$. The inner product of these two elements yields our knot invariant, which is again a sum of torsions computed at a finite number of points of $\bar{S}_{1}$.

These results raise a number of questions. First, it would be interesting to know whether the knot invariants defined above could be extended to links; and if so, to determine their relation to known invariants, and in particular to those associated with known conformal field theories. More generally, we see that the use of a real polarization gives an interesting point of view on the conformal field theory associated to current algebra. It would be interesting to know whether real polarizations arise in connection with other conformal field theories, and whether the associated moduli spaces give rise to interesting topological invariants.

\section{A Classical Integrable System in Genus One}

Let $\left(N^{2 n}, \omega\right)$ be a compact symplectic manifold, and let $L \stackrel{p}{\longrightarrow} N^{2 n}$ be a complex line bundle, with unitary connection $\alpha$, such that $d \alpha=p^{*} \omega$; then $c_{1}(L)=[\omega]$. The system $(N, \omega, L, \alpha)$ is a classical integrable system if there exists a fibration $\pi: N \rightarrow B$ whose fibres are Lagrangian submanifolds of $N$; that is, manifolds $\pi^{-1}(b)$ of dimension $n$ such that $\left.\omega\right|_{\pi^{-1}(b)}=0$.

Now let $\bar{S}_{1}$ denote the space of equivalence classes of representations $\varrho: \pi_{1}\left(\Sigma^{1}\right) \rightarrow G$, where $\Sigma^{1}$ is a two-manifold of genus one and $G$ is a compact, simple group. It is well-known that the space $\bar{S}_{1}$ is equipped with a 2 -form $\omega$ which is generically non-degenerate [2]. In [8] it was shown that the Chern-Simons 3-form may be used to construct a complex line bundle $\mathscr{L} \stackrel{p}{\longrightarrow} \bar{S}_{1}$, with a unitary connection $\alpha$, so that generically $d \alpha=p^{*} \omega$. Furthermore, in [10] we constructed a fibration $\mathscr{F}: \bar{S}_{1} \rightarrow B_{1}$ whose fibres were Lagrangian subvarieties of $\bar{S}_{1}$. Hence we have the basic elements described above for classical integrable systems - except for the degeneracy of $\omega$ and the corresponding degeneracy of the fibres of $\mathscr{F}$. For 
our purposes however it will be convenient to have a more concrete description of this integrable system, which can be obtained by considering some integrable systems associated to line bundles on complex tori. This description is self contained; in Proposition 2.1 we show it indeed corresponds to the structure of [8] and $[10]$.

\subsection{Line Bundles on Complex Tori}

Let $U \subset \mathbb{R}^{n}$ be a lattice, and let $U^{\mathbb{C}}=U \otimes \mathbb{C} \subset \mathbb{R}^{n} \otimes \mathbb{C}=\mathbb{C}^{n}$. Let there be given a nondegenerate, skew symmetric real form $E: \mathbb{C}^{n} \times \mathbb{C}^{n} \rightarrow \mathbb{R}$, which is integral on $U^{\mathbb{C}} \times U^{\mathbb{C}}$; we may as well take $\left.E\right|_{\mathbb{R}^{n} \times \mathbb{R}^{n}}=0$. Let $\alpha: U^{\mathbb{C}} \rightarrow S^{1} \hookrightarrow \mathbb{C}$ satisfy

$$
\alpha\left(u_{1}+u_{2}\right)=e^{i \pi E\left(u_{1}, u_{2}\right)} \alpha\left(u_{1}\right) \alpha\left(u_{2}\right) .
$$

Then, following Mumford [7], we define a line bundle $L(E, \alpha)$ on the complex torus $\mathbb{C}^{n} / U^{\mathbb{C}}$ as the quotient of $\mathbb{C}^{n} \times \mathbb{C}$ by the action of $U^{\mathbb{C}}$ given by

$$
\phi_{u}(z, \lambda)=\left(z+u, \alpha(u) e^{i \pi E(z, u)} \lambda\right) .
$$

The metric on $L(E, \alpha)$ is simply

$$
\|\varphi(z)\|^{2}=|\varphi(z)|^{2},
$$

which is clearly invariant under the action of $U^{\mathbb{C}}$. (Note that in [7] the metric is given by $\|\varphi(z)\|_{\theta}^{2}=e^{(\pi / 2) \operatorname{Re} H(z, z)}\|\varphi(z)\|$, where $H(z, y)=E(x, i y)=i E(x, y)$; this accounts for the apparent discrepancy in (2.2).) This metric gives rise to a unitary connection $A$ on $L(E, \alpha)$, whose curvature is precisely the constant 2 -form $\tilde{\omega}$ given by the skewsymmetric bilinear form $E$. To obtain a classical integrable system, we let $\pi: \mathbb{C}^{n} / U^{\mathbb{C}} \rightarrow \mathbb{R}^{n} / U$ be the projection onto the real part; that is, $\pi$ is the map induced on $\mathbb{C}^{n} / U^{\mathbb{C}}$ by the map $\tilde{\pi}: \mathbb{C}^{n} \rightarrow \mathbb{R}^{n}$ given by

$$
\tilde{\pi}\left(z_{1}, \ldots, z_{n}\right)=\left(\operatorname{Im} z_{1}, \ldots, \operatorname{Im} z_{n}\right) .
$$

It is clear that the fibres of $\pi$ are of dimension $n$, and since $\left.E\right|_{\mathbb{R}^{n} \times \mathbb{R}^{n}}=0$, we have $\left.\tilde{\omega}\right|_{\pi^{-1}(b)}=0$. Hence the system $\left(\mathbb{C}^{n} / U^{\mathbb{C}}, E, L(E, \alpha), A\right)$, with the fibration $\pi$, is a classical integrable system.

\subsection{Cartan Tori of Lie Groups}

Now let $G$ be a compact, simple Lie group and let $T \subset G$ be a Cartan torus. We will use the construction of Sect. 2.1 to produce an integrable system on $T \times T$.

First, by exponentiation, it is clear that

$$
T \times T \simeq t^{\mathbb{C}} / U^{\mathbb{C}},
$$

where $t^{\mathbb{C}}$ is the complexification of $t=\operatorname{Lie}(T)$, and $U^{\mathbb{C}}$ is the complexification of the root lattice $U \subset t$. Now on $t$ there exists the natural inner product $\langle,\rangle_{G}$ appropriate to the root system of $G$. This inner product is integral on $U \times U$; if $G$ is simply laced, the inner product is even on $U \times U$. We use $\langle,\rangle_{G}$ to produce a skew symmetric real form $E_{G}: t^{\mathbb{C}} \times t^{\mathbb{C}} \rightarrow \mathbb{R}$ by letting

$$
E_{G}\left(p+i q, p^{\prime}+i q^{\prime}\right)=\left\langle p, q^{\prime}\right\rangle_{G}-\left\langle q, p^{\prime}\right\rangle_{G} .
$$


Now suppose $G$ is simply laced; we may then choose $\alpha \equiv 1$, and obtain a line bundle $L\left(E_{G}, 1\right) \rightarrow T \times T$, with a unitary connection whose curvature is $E_{G}$. Likewise we have a fibration $\tilde{\mathscr{F}}: T \times T \rightarrow T$ whose fibres are Lagrangian submanifolds of $T \times T$.

\subsection{The Space $\bar{S}_{1}$}

Now recall that $\bar{S}_{1}$ was the space of equivalence classes of representations $\varrho: \pi_{1}\left(\Sigma^{1}\right)$ $=Z \oplus Z \rightarrow G$. Any such representation is in fact a representation $\varrho: Z \oplus Z \rightarrow T$, where $T$ is a Cartan torus of $G$. Since all such tori are isomorphic, $\bar{S}_{1}$ may be described by

$$
\bar{S}_{1} \simeq T \times T / W_{\text {diag }},
$$

where $W_{\text {diag }}$ is the Weyl group of $G$, acting diagonally on $T \times T$. It was on this space that were defined the symplectic form $\omega$, the line bundle $\mathscr{L}$, and the fibration $\mathscr{F}$ described in [8] and [10]. On the other hand we have the following result. Let $\pi: T \times T \rightarrow(T \times T) / W, \hat{\pi}: T \rightarrow T / W$ denote the projections.

Proposition 2.1. (i) There exists a line bundle $\hat{\mathscr{L}} \rightarrow T \times T / W$ such that $\pi^{*} \hat{\mathscr{L}}$ $=L\left(E_{G}, 1\right)$.

(ii) There exists a function $\hat{\mathscr{F}}:(T \times T) / W \rightarrow T$ such that the following square commutes

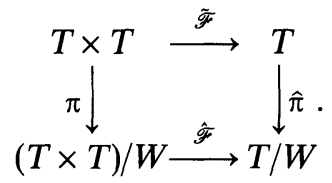

(iii) Let $\mathscr{L} \rightarrow T \times T / W$ denote the line bundle of [8]; then $\mathscr{L}=\hat{\mathscr{L}}$.

(iv) Let $\mathscr{F}: T \times T / W \rightarrow T / W$ denote the fibration of [10]. Then $\hat{\mathscr{F}}=\mathscr{F}$.

Proof. Parts (i) and (ii) are immediate: The existence of the line bundle $\hat{\mathscr{L}}$ follows since the inner product $\langle,\rangle_{G}$, and hence the skew form $E_{G}$, is $W$-invariant. Likewise $\hat{\mathscr{F}}$ exists since $\hat{\pi} \circ \hat{\mathscr{F}}(x)=\hat{\pi} \circ \hat{\mathscr{F}}(y)$ whenever $\pi(x)=\pi(y)$. It remains to show (iii) and (iv). (iii) Let $\mathscr{A}_{F}$ denote the space of flat $G$ connections on $\Sigma^{1}$, and $\mathscr{G}=\operatorname{Maps}\left(\Sigma^{1}, G\right)$ the group of gauge transformations. Then $\mathscr{A}_{F} / \mathscr{G} \cong T \times T / W$, and by the results of [8], the bundle $\mathscr{L} \rightarrow \mathscr{A}_{F} / \mathscr{G}$ is given by the unitary cocycle $\theta: \mathscr{A}_{F} \times \mathscr{G} \rightarrow S^{1}$ given by

$$
\theta(A, g)=\exp \frac{i}{4 \pi} \operatorname{tr} \int_{\Sigma^{1}} A g^{-1} d g+\frac{i}{12 \pi} \operatorname{tr} \int_{Y}\left(\tilde{g}^{-1} d \tilde{g}\right)^{3},
$$

where $Y$ is any 3-manifold whose boundary is $\Sigma$, and $\tilde{g}$ is an arbitrary extension of $g$ to $Y$. On the space $\mathscr{A}_{F}^{C}=T \times T$ of constant gauge fields, the remnant of the gauge group is precisely $U^{\mathbb{C}} \times W$; then $\widetilde{S}_{1}=\mathscr{A}_{F}^{\mathbb{C}} / U^{\mathbb{C}} \times W$. For $g \in U^{\mathbb{C}}$, we may choose $Y$ so that $g$ extends to $Y$ as a map $\tilde{g}: Y \rightarrow T$; then the second term vanishes, and

$$
\theta(A, g)=\exp \pi i E_{G}(x, u),
$$

where $x \in T \times T$ corresponds to $A$ and $u \in U^{\mathbb{C}}$ corresponds to $g$. For $g \in W$, we may choose $g$ and $\tilde{g}$ to be constant; then $\theta(A, g)=1$. Hence the line bundle $\mathscr{L}$ coincides precisely with $\hat{\mathscr{L}}$. 
Remark. The line bundle $\mathscr{L}$ exists by [8] for any group $G$, and $\pi^{*} \mathscr{L}$ must be of the form $L\left(E_{G}, \alpha\right)$ for some $\alpha$. However, if $G$ is not simply laced, $E_{G}$ is not even, and $\alpha=1$ is not a solution of (2.2). It would be interesting to construct $\alpha$ in this case.

(iv) We recall the construction of the fibration $\mathscr{F}: \bar{S}_{1} \rightarrow B_{1}$. First, $B_{1}$ was defined quotient of $G$ by the adjoint action of $G$ on itself; it is clear that

$$
B_{1}=G / G_{\mathrm{adj}}=T / W \text {. }
$$

Second, we had $\bar{R}_{1} \stackrel{i}{\hookrightarrow} G \times G$ as the zero set of the function $R(a, b)=a b a^{-1} b^{-1}-1$. Then the fibration $\mathscr{F}$ was the map $\mathscr{F}: \bar{S}_{1} \rightarrow B_{1}$ induced by the projection onto the first factor

$$
\pi_{1}: G \times G \rightarrow G
$$

and completing the diagram

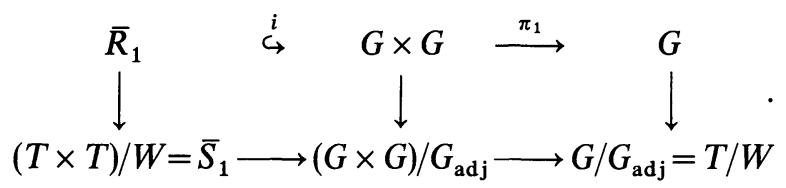

It is then clear that the following square commutes

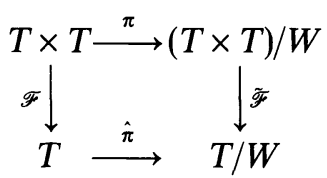

proving (iv).

\section{The Quantized System in Genus 1}

Let $\left(N^{2 n}, \omega, L, \alpha\right)$, along with a fibration $\pi: N^{2 n} \rightarrow B$, be a classical integrable system as described in Sect. II. Let $S_{\pi}$ denote the sheaf of local sections of $L$ which are covariantly constant along the fibres of $\pi$. The quantization of this system is then the vector space $H^{*}\left(N, S_{\pi}\right)$, which, as we shall see below, may be provided with a natural Hilbert space structure.

In [11] Sniatycki has shown that the sheaf cohomology $H^{*}\left(N, S_{\pi}\right)$ may be computed explicitly. We summarize his results in Proposition 3.1. Let $b \in B$, and let $r_{b}: \pi^{-1}(b) \rightarrow N$ be the inclusion. The fibre $\pi^{-1}(b)$ is said to satisfy the BohrSommerfeld condition if the holonomy of $r_{b}^{*} \alpha$ about any closed loop in $\pi^{-1}(b)$ is trivial. Since the curvature of $r_{b}^{*} \alpha$ is zero, any such fibre is equipped with a global covariant constant section $s_{b}: \pi^{-1}(b) \rightarrow r_{b}^{*} L$, unique up to a constant multiple. Let $B_{b s}$ denote the set of points $b \in B$ such that $\pi^{-1}(b)$ satisfies the Bohr-Sommerfeld condition; for $b \in B_{b s}$, let $S_{b}$ denote the one dimensional vector space generated by $s_{b}$.

Proposition 3.1 (Sniatycki) [11].

(i) $H^{i}\left(N^{2 n}, S_{\pi}\right)=0$ if $i \neq n$.

(ii) There exists a natural isomorphism $H^{n}\left(N^{2 n}, S_{\pi}\right) \simeq \oplus_{b \in B_{b s}} S_{b}$. 
Now the space $H^{n}\left(N, S_{\pi}\right)$ has a natural Hilbert space structure given by the inner product

$$
\begin{gathered}
\left\langle s_{b}, s_{b^{\prime}}\right\rangle=0, \quad b \neq b^{\prime}, \\
\left\langle s_{b}, s_{b}\right\rangle=\left\|s_{b}(x)\right\|^{2},
\end{gathered}
$$

where $x \in \pi^{-1}(b)$ is arbitrary; since $s_{b}$ is covariant constant, the inner product is independent of the point $x$. Heuristically the sections $s_{b}$ may be looked upon as distributional sections of $L$ concentrated on $\pi^{-1}(b)$.

\subsection{Quantization on Complex Tori}

We may apply Sniatycki's method to the complex torus $\mathbb{C}^{n} / U^{\mathbb{C}}$, with line bundle $L(E, \alpha)$ and fibration $\pi: \mathbb{C}^{n} / U^{\mathbb{C}} \rightarrow \mathbb{R}^{n} / U$. The Bohr-Sommerfeld condition for the fibre $\pi^{-1}(b)$ then reads

$$
\alpha(u) e^{i \pi E(z, u)}=1
$$

for all $z \in \pi^{-1}(b), u \in U$.

Now for $u \in U$, we may choose a linear functional $\lambda \in\left(\mathbb{C}^{n}\right)^{*}$, real on $\mathbb{R}^{n} \subset \mathbb{C}^{n}$, such that

$$
\alpha(u)=\exp i \pi \lambda(u)
$$

for all $u \in U$.

In terms of $\lambda,(3.2)$ reads

$$
\lambda(u)+E(b, u) \in 2 \mathbb{Z}
$$

for all $u \in U$. Thus there will be precisely $\sqrt{\operatorname{det} E}$ points in $\left(\mathbb{R}^{n} / U\right)_{b s}$; and the global covariant constant section on the fibre $\pi^{-1}(b)$ is simply

$$
s_{b}(z)=\exp i \pi E(b, z)+\pi i \lambda(z) .
$$

The above considerations take a particularly simple form for the complex torus $t^{\mathbb{C}} / U^{\mathbb{C}}$, and line bundle $\left(L\left(E_{G}, 1\right)\right)^{\otimes k}$, considered in Sect. 2.2 . In this case the BohrSommerfeld fibres are those located at points $b \in t / U$ satisfying

$$
k E(b, u)=k\langle b, u\rangle \in 2 \mathbb{Z}
$$

for $u \in U$; that is

$$
(t / U)_{b s}=\frac{1}{k} \cdot U .
$$

The section $s_{b}$ at the point $\frac{1}{k} \cdot u=b$ is simply

$$
s_{b, k}(z)=\exp i \pi k<\frac{1}{k} \cdot u, \operatorname{Re} z>
$$

\subsection{Relation to $\Theta-$ Functions}

The dimension $\sqrt{\operatorname{det} E}$ of the cohomology $H^{n}\left(\mathbb{C}^{n} / U^{\mathbb{C}}, S_{\pi}\right)$ is precisely that of the space of $\theta$-functions associated to the torus $\mathbb{C}^{n} / U^{\mathbb{C}}$ and line bundle $L(E, \alpha)$, and 
obtained by quantizing this symplectic manifold in a Kähler polarization. In fact the isomorphism between the two spaces may be given explicitly. Let us do this for the case of the complex torus $t^{\mathbb{C}} / U^{\mathbb{C}}$. $[7,6]$

The holomorphic sections $\widetilde{\theta}(z)$ of the bundle $\left(L\left(E_{G}, 1\right)\right)^{\otimes k} \rightarrow t^{\mathbb{C}} / U^{\mathbb{C}}$ are given by

$$
\tilde{\theta}_{\gamma / k, k}(z)=\exp \left(\frac{\pi k z^{2}}{2}\right) \cdot \sum_{\alpha \in U} e^{-\pi k(\alpha+\gamma / k)^{2}+2 \pi i k(\alpha+\gamma / k) z}
$$

for $\gamma \in U$.

Now recall that these sections are defined with respect to the standard metric on the line bundle $\left(L\left(E_{G}, 1\right)\right)^{\otimes k}$, which is

$$
\|S(z)\|^{2}=e^{-\pi k|z|^{2}}|S(z)|^{2} .
$$

Thus, to compare them to our sections, we write

$$
\hat{\theta}_{\gamma / k, k}(z)=e^{\pi k|z|^{2} / 2} \widetilde{\theta}_{\gamma / k, k}(z) .
$$

A computation establishes the following result.

Proposition 3.2. Let $\gamma, \gamma^{\prime} \in U$. Then

$$
\int_{\hat{\mathscr{F}}-1(\gamma / k)} s_{\gamma / k, k}(z)^{*} \hat{\theta}_{\gamma^{\prime} / k, k}(z)=C \cdot \delta_{\gamma \gamma^{\prime}}
$$

where $C>0$. Hence the quantization of the classical system is seen to be independent of the polarization.

\subsection{Quantization on $\bar{S}_{1}$}

We now turn to the quantization of the integrable system constructed on $\bar{S}_{1}=T \times T / W$. In this case, due to the degeneracies of the fibres of $\mathscr{F}$, we are not able to apply Sniatycki's theorem. This is best illustrated in the case $G=S U(2)$ (see Fig. 1). In this case the moduli space is a two-torus with identification of points differing by reflection about the origin; in the figure, each point on an edge of the triangle shown is identified with its image under reflection about the midpoint of that edge. The Bohr-Sommerfeld subvarieties (shown here for $k=3$ ) are located at $y=0 / 2 k, 1 / 2 k, \ldots, k / 2 k$. Most of these subvarieties are circles (because of the edge identifications). The exceptions are the degenerate fibres at 0 and $1 / 2$, which are copies of the interval $[0,1]$.

We quantize this system as follows. The sheaf $S_{\mathscr{F}}$ is defined as the sheaf of local sections of the line bundle $\mathscr{L}$ which are continuous everywhere, smooth (with respect to the smooth structure obtained from the smooth structure on $T \times T$ ) away from the fixed points of $W$, and locally covariantly constant at the nondegenerate fibres of $\mathscr{F}$. Such sections automatically come from $W$ invariant sections of $S_{\mathscr{F}}$ on $T \times T$. Hence we obtain the following result.

\section{Proposition 3.3.}

(i) $H^{i}\left(T \times T / W, S_{\mathscr{F}}\right)=0$ if $i \neq n$.

(ii) There exists a natural isomorphism $H^{n}\left(T \times T / W, S_{\mathscr{F}}\right) \simeq \oplus_{b \in \frac{1}{k} \cdot U_{b}^{-}}$.

Here $S_{b}^{-}$is the one dimensional vector space generated by the section

$$
s_{b}^{-}=\sum_{w \in W}(-1)^{w} s_{w b}
$$




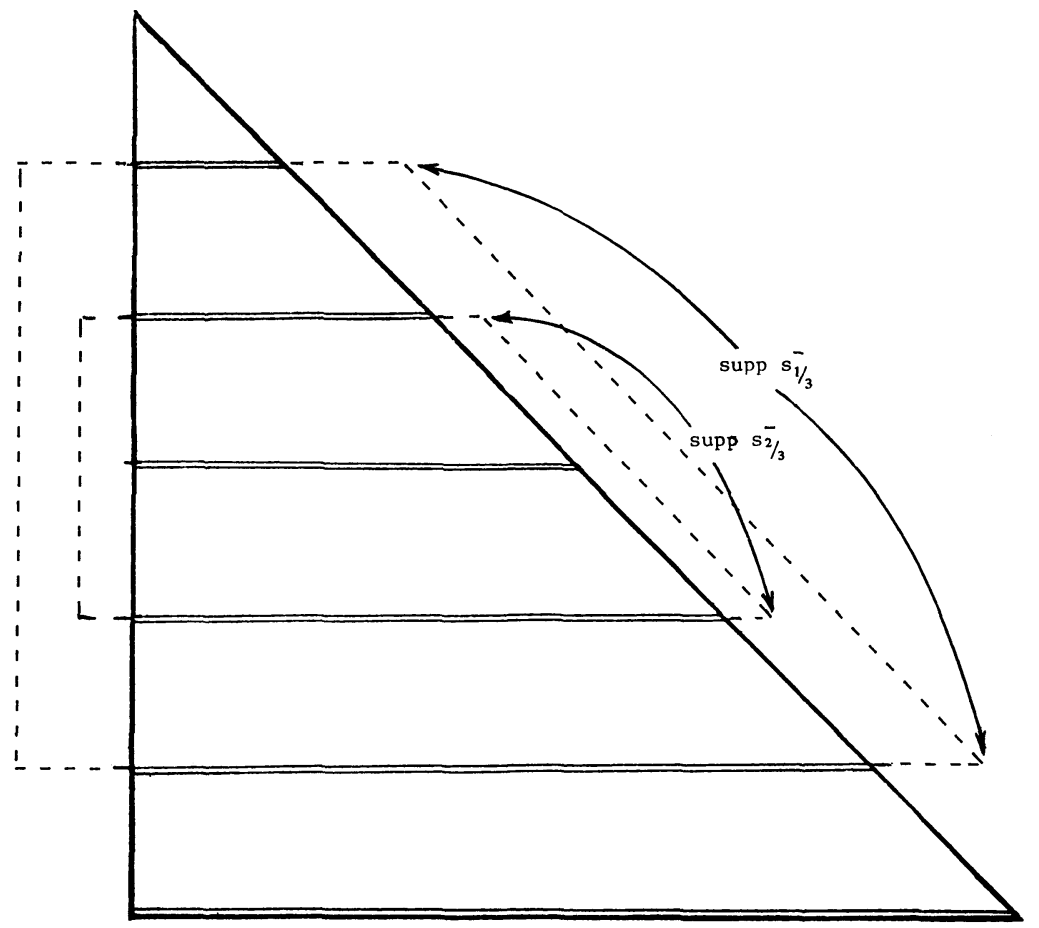

Fig. 1. The moduli space $\overline{S_{1}}$ for $G=S U(2)$, along with the Bohr-Sommerfeld fibers of $\mathscr{F}$ in the case $k=3$. The fibers marked supp $s_{1 / 3}^{-}$and supps $s_{2 / 3}^{-}$are circles, and correspond to elements of $H^{*}\left(\bar{S}_{1}, S_{\mathscr{F}}\right)$. The other two fibers are copies of the interval $[0,1]$

where, for $w \in W$,

$$
s_{w b}(x)=s_{b}(w x), \quad x \in \pi^{-1}(w b) .
$$

The space $H^{n}\left(T \times T / W, S_{\mathscr{F}}\right)$ is clearly a Hilbert space, with the inner product induced by the inner product on $H^{n}\left(T \times T, S_{\tilde{F}}\right)$. To compute its dimension, we first remark that some of the sections $s_{b}^{-}$are zero; these sections correspond to degenerate fibres of $\mathscr{F}$. Those which remain are in one to one correspondence with the set $P=\left\{\varrho+\frac{1}{k} \cdot \gamma\right\}$, where $\varrho$ is one half the sum of the positive roots of $G$, and $\gamma$ is a positive weight of $G$. The cardinality of $P$ is in fact precisely the number of integrable, level $k$, representations of the affine lie algebra $\hat{G}$. This is more than a coincidence; the Weyl-Kac characters of these representations are related to the $\theta$-functions by

$$
\chi_{\lambda+\varrho, k+h}=\frac{1}{\Pi} \theta_{\lambda+\varrho, k+h}^{-} \equiv \frac{1}{\Pi} \sum(-1)^{w} \theta_{w(\lambda+\varrho), k+h},
$$

where $\theta_{\lambda+\varrho, k+h}=\exp -\left(\pi(k+h) \frac{z^{2}}{2}\right) \tilde{\theta}_{\lambda+\varrho, k+h}$ and $\Pi(z)=\theta_{\varrho, h}^{-}(z)$.

As a consequence of Proposition 3.2, we have the following 
Corollary 3.4. Let $\lambda, \lambda^{\prime} \in \frac{1}{k+h} \cdot U_{+}$, where $U_{+}$is the intersection of $U$ with the positive Weyl chamber. Then

$$
\int_{\mathscr{F}^{-1}\left(\lambda^{\prime}+\varrho\right)} \chi_{\lambda+\varrho, k+h} \cdot \Pi \cdot \exp \pi(k+h) \frac{z^{2}}{2} \cdot \exp \pi(k+h) \frac{|z|^{2}}{2} \cdot s_{\lambda^{\prime}+\varrho, k+h}{ }^{*}=\text { const } \cdot \delta_{\lambda \lambda^{\prime}} .
$$

In conformal field theory the characters $\chi_{\lambda+\varrho, k+h}$ appear as conformal blocks of the current algebra of $G$ at what is denoted as level $k$ [4]. We see here that they are related to the quantization of the line bundle $\mathscr{L}^{\otimes(k+h)}$, where $k \geqq 0$. The shift $k \rightarrow k+h$ will reappear in our computations of manifold invariants in Sect. V. It would be interesting to know what role the $s_{\lambda+e}^{-}$play in the representation theory of affine Lie algebras.

\section{Action of the Modular Group}

The construction of the quantum Hilbert spaces $H^{n}\left(\bar{S}_{1}, S_{\mathscr{F}}\right)$ involved the choice of a polarization $\mathscr{F}$ of $\bar{S}_{1}$; and this, in turn, depend upon a choice of generators for $\pi_{1}\left(\Sigma^{1}\right)$. This choice cannot be fixed by diffeomorphisms of $\Sigma^{1}$; so we must now turn to investigating the behavior of $H^{n}\left(\bar{S}_{1}, S_{\mathscr{F}}\right)$ under the diffeomorphism group.

First, it is clear that on the symplectic vector space $H_{1}\left(\Sigma^{1}, \mathbb{Z}\right)$, the map $\phi_{*}$ induced by a diffeomorphism $\phi: \Sigma^{1} \rightarrow \Sigma^{1}$ is a linear symplectic map; in other words, an element of $S L(2, \mathbb{Z})$. As generators of this group we choose $S=\left(\begin{array}{cc}0 & 1 \\ -1 & 0\end{array}\right), T=\left(\begin{array}{ll}1 & 1 \\ 0 & 1\end{array}\right)$. The action of $S L(2, \mathbb{Z})$ on $T \times T$ is the induced action on $H_{1}\left(\Sigma^{1}, T\right)$. This action can be described explicitly as the action induced on $T \times T$ by the following action of $S$ and $T$ on the complexified torus $t^{\mathbb{C}}$ :

$$
\begin{aligned}
& T\left(\left(z_{1}, \ldots, z_{n}\right)\right)=\left(z_{1}+\operatorname{Im} z_{1}, \ldots, z_{n}+\operatorname{Im} z_{n}\right), \\
& S\left(\left(z_{1}, \ldots, z_{n}\right)\right)=\left(z_{i} / i, \ldots, z_{n} / i\right) .
\end{aligned}
$$

As is plain from (4.1), the generator $T$ preserves the polarization $\tilde{\mathscr{F}}$, whereas the generator $S$ does not. So we must review the construction of the induced group action in each of these cases.

\subsection{Group Actions and Quantization}

Let $\left(N^{2 n}, \omega, L, \alpha\right)$, along with a fibration $\pi$, be a classical integrable system, and let $H^{n}\left(N, S_{\pi}\right)$ be its quantization. Suppose we are given a transformation $G: N \rightarrow N$ such that $G^{*} L=L$ as line bundles with unitary connections. Let us first consider the case where $G$ preserves the polarization $\pi$; that is, suppose

$$
\pi(G(x))=\pi(x)
$$

for all $x \in N$.

Let us choose a basis $\left\{s_{b}\right\}_{b \in B_{b s}}$ for $H^{n}\left(N, S_{\pi}\right)$, as provided for by Sniatycki's theorem (Proposition 3.1). We define the induced action of $G$ on $H^{n}\left(N, S_{\pi}\right)$ by

$$
\left(G_{*} s_{b}\right)(x)=s_{b}(G(x))=\gamma_{b} s_{b}(x)
$$

It is then clear that, given a group of such transformations, the induced action on $H^{n}\left(N, S_{\pi}\right)$ results in a representation of this group. 
On the other hand, suppose $G: N \rightarrow N$ is a symplectomorphism, preserving $L$ and $\alpha$ as before, but not preserving $\pi$. Suppose instead that the fibres of the map $\pi_{G}=\pi \circ G: N \rightarrow B$ (which are of necessity Lagrangian submanifolds) are transverse to those of $\pi$. It is then clear that a fibre $\pi^{-1}(b)$ satisfies the Bohr-Sommerfeld condition if and only if $\pi_{G}^{-1}(b)$ does. Thus we may identify the cohomology spaces $H^{n}\left(N, S_{\pi}\right)$ and $H^{n}\left(N, S_{\pi_{G}}\right)$, via a map $G_{\#}: H^{n}\left(N, S_{\pi}\right) \rightarrow H^{n}\left(N, S_{\pi_{G}}\right)$ defined by

$$
G_{\#}\left(s_{b}\right)(x)=s_{b}(G(x))
$$

for $x \in \pi_{G}^{-1}(b)$.

We use this map to produce an action of $G$ on $H^{n}\left(N, S_{\pi}\right)$ by using the BKS pairing [13], defining

$$
\left(G_{*} s_{p}\right)(x)=\sum_{q \in B_{b s}} \sum_{y \in \pi^{-1}(q) \cap \pi_{\bar{G}^{1}}(p)}\left\langle s_{q}(y), G_{\#} s_{p}(y)\right\rangle \cdot s_{q}(x),
$$

where the sums in question are finite since the fibres of $\pi_{G}$ intersected those of $\pi$ transversely.

If we are given a group of transformations of this type, there is no assurance that this pairing will indeed produce a representation. This is however the case in many examples; as we shall see, the action of $S L(2, \mathbb{Z})$ on the complex tori (and on $\left.\bar{S}_{1}\right)$ fit into this category.

\subsection{The $S L(2, \mathbb{Z})$ Action on the Torus}

It is now straightforward to compute the induced actions on $S, T$ on $H^{n}\left(T \times T, S_{\tilde{F}}\right)$. We have the following result.

Proposition 4.1. The induced actions of the symplectomorphisms $S, T$ on $H^{n}\left(\mathbb{C}^{n} / U^{\mathbb{C}}, S_{\tilde{F}}\right)$ are given by

$$
\begin{aligned}
& \left(\widetilde{T}_{*}\right)_{\frac{1}{k} u, \frac{1}{k} v}=\delta_{u v} \exp (i \pi\langle u, v\rangle / 2 k), \\
& \left(\widetilde{S}_{*}\right)_{\frac{1}{k} u, \frac{1}{k} v}=\exp (i \pi\langle u, v\rangle / k)
\end{aligned}
$$

for $u, v \in U$. The matrices $\widetilde{S}_{*}, \widetilde{T}_{*}$ induce a representation of the group $S L(2, \mathbb{Z})$ on $H^{n}\left(T \times T, S_{\tilde{F}}\right)$.

To compute the induced action on $H^{n}\left(\bar{S}_{1}, S_{\mathscr{F}}\right)$, we use the description in Proposition 3.3 of the elements of $H^{n}\left(\bar{S}_{1}, S_{\mathscr{F}}\right)$ as $W$-invariant elements of $H^{n}\left(T \times T, S_{\mathscr{F}}\right)$. We then have the following result:

Proposition 4.2. The induced action of the symplectomorphisms $S, T$ on the space $\bar{S}_{1}$ are given by

$$
\begin{aligned}
& \left(T_{*}\right) \frac{1}{k} u, \frac{1}{k} v=\delta_{u v} \exp (i \pi\langle v, v\rangle / 2 k), \\
& \left(\bar{S}_{*}\right) \frac{1}{k} u, \frac{1}{k} v=\sum_{w \in W}(-1)^{w} \exp (i \pi\langle u, v\rangle / k)
\end{aligned}
$$

for $\frac{1}{k} u, \frac{1}{k} v \in\left\{\varrho+\frac{1}{k} U\right\}$. The matrices $\left(T_{*}\right), \frac{1}{\sqrt{k}} \frac{1}{|W|} \bar{S}_{*}=S_{*}$ induce a representation of $S L(2, \mathbb{Z})$ on $H^{n}\left(\bar{S}_{1}, S_{\mathscr{F}}\right)$. 
Remark. Let $G=S U(2)$. Then, for $k \geqq 2$, we have

$$
\begin{aligned}
& \left(T_{*}\right)_{p / k, q / k}=\delta_{p, q} \exp \frac{i \pi}{2 k} p^{2}, \\
& \left(S_{*}\right)_{p / k, q / k}=\frac{1}{\sqrt{k}} \sin \pi p q / k .
\end{aligned}
$$

These are the results of [6]. In general the representations described here are (not surprisingly) simply the representations of the modular group appearing in the theory of level- $k \theta$-functions.

The proofs of Propositions 4.1 and 4.2 are simple computations; to verify that the relevant matrices induce representations of $S L(2, \mathbb{Z})$ it suffices to check that $S_{*}^{2}=-1,\left(S_{*} T_{*}\right)^{3}=1$. This follows immediately from the formulas.

\section{Topological Quantum Field Theory}

\subsection{Axioms for Topological Field Theory}

We now wish to try to use our construction to obtain a topological quantum field theory in dimension 3 - or as much of it as we can. Let us recall the axioms for topological field theory [1]. A topological quantum field theory is an assignment $Z$ of smooth oriented 2-manifolds to finite dimensional Hilbert spaces and diffeomorphisms to linear isomorphisms satisfying the following axioms:

1. If $\Sigma^{*}$ denotes the smooth manifold $\Sigma$ with orientation reversed, there exists a canonical isomorphism

$$
(Z(\Sigma))^{*} \simeq Z\left(\Sigma^{*}\right)
$$

where $(Z(\Sigma))^{*}$ denotes the dual vector space.

2. If $\Sigma_{1} \cup \Sigma_{2}$ is a disjoint union, then

$$
Z\left(\Sigma_{1} \cup \Sigma_{2}\right)=Z\left(\Sigma_{1}\right) \otimes Z\left(\Sigma_{2}\right) .
$$

3. Let $Y^{3}$ be a smooth, oriented 3-manifold with boundary $\partial Y^{3}=\Sigma$. Then there is an element $V(Y) \in Z(\Sigma)$; if $\Phi: Y^{3} \rightarrow Y^{3^{\prime}}$ is a diffeomorphism of pairs,

$$
V\left(Y^{3^{\prime}}\right)=Z\left(\left.\Phi\right|_{\partial Y^{3}}\right)\left(V\left(Y^{3}\right)\right) \in Z\left(\partial Y^{3^{\prime}}\right) .
$$

4. Let $\partial Y^{3}=\Sigma_{1} \cup \Sigma_{2}, \partial Y^{3^{\prime}}=\Sigma_{2}^{*} \cup \Sigma_{3}$. We may consider

$$
V\left(Y^{3}\right) \in \operatorname{Hom}\left(Z\left(\Sigma_{1}\right)^{*}, Z\left(\Sigma_{2}\right)\right), \quad V\left(Y^{3}\right) \in \operatorname{Hom}\left(Z\left(\Sigma_{2}\right), Z\left(\Sigma_{3}\right)\right),
$$

and

$$
V\left(Y^{3} \cup_{\Sigma_{2}} Y^{3^{\prime}}\right) \in \operatorname{Hom}\left(Z\left(\Sigma_{1}\right)^{*}, Z\left(\Sigma_{3}\right)\right)
$$

then, as linear maps,

$$
V\left(Y^{3} \cup Y^{3^{\prime}}\right)=V\left(Y^{3^{\prime}}\right) \circ V\left(Y^{3}\right) .
$$

5. Let $Y^{3^{*}}$ denote $Y^{3}$ with opposite orientation. Then $V\left(Y^{3^{*}}\right)=\left\langle V\left(Y^{3}\right), \cdot\right\rangle$, where $\langle$,$\rangle is the inner product on Z\left(\Sigma=\partial Y^{3}\right)$.

6. The set $\left\{V\left(Y^{3}\right) \mid \partial Y^{3}=\Sigma\right\}$ spans $Z(\Sigma)$.

As a consequence of these axioms, we have the assignment of an invariant $V\left(M^{3}\right) \in \mathbb{C}$ to a three manifold $M^{3}$ with empty boundary. And if we write 
$M^{3}=Y^{3} \cup_{\Sigma} Y^{3^{\prime}}$, where $Y^{3}$ and $Y^{3^{\prime}}$ are three manifolds with boundary $\Sigma$, then $V\left(M^{3}\right)=\left\langle V\left(Y^{3}\right), V\left(Y^{3^{\prime}}\right)\right\rangle$. It remains to produce an example of the assignments $Z$ and $V$.

Now let $\Sigma^{g}$ be a Riemann surface of genus $g$. Then the space $\bar{S}_{g}$ of representations $\varrho: \pi_{1}\left(\Sigma^{g}\right) \rightarrow G$ contains a large open cell $S_{g}$ which is a Kahler manifold; and the line bundle $\mathscr{L} \rightarrow S_{g}$ is a holomorphic line bundle. Then Witten has proposed [12] that the assignment $\Sigma^{g} \rightarrow H_{\mathrm{hol}}^{0}\left(\bar{S}_{g}, \mathscr{L}\right)$ induces a topological quantum field theory. For this it is necessary to show that this assignment is independent of choice of holomorphic structure; this was done in $[3,9]$. What has not however been provided is a geometric prescription for the assignment $V(Y) \in Z(\Sigma)$ for $Y$ such that $\partial Y=\Sigma$. We propose here a different construction of the space $Z(\Sigma)$ which will enable us to make some progress towards constructing the element $V(Y)$.

Specifically, we suggest that the vector space $Z(\Sigma)$ be taken to be

$$
Z\left(\Sigma^{g}\right)=H^{*}\left(\bar{S}_{g}, S_{\mathscr{F}}\right),
$$

where $\mathscr{F}$ is the foliation of [10], and $S_{\mathscr{F}}$ is the sheaf of local sections of $\mathscr{L} \rightarrow \bar{S}_{g}$ which are covariantly constant along the nondegenerate fibres of $\mathscr{F}$, smooth on $S_{g}$, and continuous everywhere. We now turn to see how this works in genus 1.

\subsection{Topological Quantum Field Theory in Genus 1}

In this case the space $H^{*}\left(\bar{S}_{1}, S_{\mathscr{F}}\right)$ has been shown to be isomorphic to $H_{\mathrm{hol}}^{*}\left(\bar{S}_{1}, \mathscr{L}\right)$, and hence our construction coincides with Witten's proposal.

However, the specific use of the polarization $\mathscr{F}$ allows us to make a guess concerning the assignment $Y \rightarrow V(Y)$. Given a presentation $\partial Y=\Sigma$, we obtain a natural isotropic subvariety $L_{Y}$ of $\bar{S}_{q}$, given by those representations $\varrho: \pi_{1}\left(\Sigma^{g}\right) \rightarrow G$ which extend to representations $\tilde{\varrho}: \pi_{1}(Y) \rightarrow G$. We will from now on concentrate on the case where this isotropic space is maximal, and therefore Lagrangian. In the case $g=1$, the space $L_{Y}$ is the image of $\bar{S}_{1}$ of a Lagrangian vector subspace of $H_{1}\left(\Sigma^{1}, \mathbb{R}\right)$. Any such Lagrangian vector subspace is related, via an $S L(2, \mathbb{Z})$ transformation $\psi_{Y}$, to the "standard" Lagrangian vector subspace associated with the presentation of $\Sigma$ as the boundary of the solid torus $S^{1} \times D^{2}$. Hence if we can produce an element $V\left(S^{1} \times D^{2}\right) \in Z(\Sigma)$, we will have an element $V(Y) \in Z(\Sigma)$, defined by

$$
V(Y)=\psi_{Y_{*}} V\left(S^{1} \times D^{2}\right) .
$$

We note that there will be more than one possible choice of element $\psi_{Y}$; different choices will differ by right multiplication by $T^{m}$ for some integer $m$, where $T$ is the element of the modular group described in the beginning of Sect. IV. We shall see that different choices of element $\psi_{Y}$ will correspond to different normalizations of the element $V(Y)$.

So let us consider the case where $\Sigma^{1}$ is presented as the boundary of the solid torus $S^{1} \times D^{2}$ in the standard way. It would be natural to try to assign to this three manifold an element of $Z(\Sigma)$ corresponding to the Lagrangian subvariety $L_{S^{1} \times D^{2}}$ $=\mathscr{F}^{-1}(0)$ of $\bar{S}_{1}$. Unfortunately there is no nonzero element of $Z(\Sigma)$ corresponding to this Lagrangian subvariety, which is degenerate; in other words, $0 \notin P$. Hence this first guess cannot be right.

Let us however, try instead the assignment

$$
V\left(S^{1} \times D^{2}\right)=s_{\varrho / k}^{-},
$$


where, as in Sect. 3.3, $\varrho$ is half the sum of the positive roots of $G$. This is a nonzero element of $Z(\Sigma)$. We use this element and (5.1) to define the element $V(Y)$ for any three manifold bounding $\Sigma$ (and with $L_{Y}$ Lagrangian) by Eq. (5.1) above. We note that the ambiguity in the choice of $\psi_{Y}$ gives rise to a multiplicative constant in the definition of $V(Y)$, with different choices related by powers of $\exp (i \pi / k)$, as a consequence of the formula for the action of $T$ on $Z(\Sigma)$ given in Proposition 4.2.

We can use this guess for the assignment $V(Y)$ to compute $V\left(S^{3}\right)$ and obtain a check of our results with those of Witten. To do so we form a three-sphere by gluing two solid tori via the diffeomorphism of $\Sigma=S^{1} \times S^{1}=\partial\left(S^{1} \times D^{2}\right)$ which exchanges the $S^{1}$ factors. This diffeomorphism clearly induces on $Z(\Sigma)$ the action of the element of $S L(2, \mathbb{Z})$ denoted in Sect. IV above by $S$. Therefore, $V\left(S^{3}\right)$ is given, using axiom (4) for topological field theory and the assignment (5.2), by

$$
V\left(S^{3}\right)=\left\langle s_{\varrho / k}^{-}, S_{*} s_{\varrho / k}^{-}\right\rangle=\left(S_{*}\right)_{\varrho / k, \varrho / k} .
$$

In the case $G=S U(2)$, this is equal to

$$
V\left(S^{3}\right)=\frac{1}{\sqrt{k}} \sin \left(\frac{\pi}{k}\right) .
$$

This agrees with the results of [12]; note that one must make the substitution $k \rightarrow k+2$ as in Sect. III, where we saw that the expressions corresponding to what is usually called current algebra at level $k$ arise from quantization of the line bundle $\mathscr{L}^{\otimes k+2}$. Using (5.1) and (5.2) we can now compute the putative invariants $V(M)$ for a wide class of three manifolds $M$. The computation of these invariants is particularly simple when $M$ is given by a Heegaard splitting along a torus. In this case, there is no ambiguity in the choice of element of $S L(2, \mathbb{Z})$, since the manifold $M$ is defined in terms of such an element $\psi \in S L(2, \mathbb{Z})$. Using (5.1) and (5.2), we obtain immediately the formula $V(M)=\left(\psi_{*}\right)_{\varrho / k, \varrho / k}$, which is in agreement with the results of [12].

We may perform further computations by noting that the topological quantum field theory we are considering admits the following generalization of axiom (3):

(3') Let $Y^{3}$ be a smooth, oriented 3-manifold, with boundary $\partial Y^{3}=\Sigma$. Let $L$ be a (framed) link in $Y^{3}$, and let $\lambda$ be a weight of a level- $k$ integrable representation of $\hat{G}$. Then there is an element $V_{\lambda}\left(Y^{3}, L\right) \in Z(\Sigma)$; if $\Phi:\left(Y^{3}, L\right) \rightarrow\left(Y^{3^{\prime}}, L^{\prime}\right)$ is a diffeomorphism of pairs,

$$
V_{\lambda}\left(Y^{3^{\prime}}, L^{\prime}\right)=Z\left(\left.\Phi\right|_{\partial Y^{3}}\right)\left(V_{\lambda}\left(Y^{3}, L\right)\right) \text {. }
$$

So let us generalize our guess for the assignment $V$ above by assigning to the solid torus $S^{1} \times D^{2}$, with an unlinked circle in marked by a representation $\lambda$ along its meridian, the element $s_{(\varrho+\lambda) / k}^{-} \in H^{*}\left(\bar{S}_{1}, S_{\mathscr{F}}\right)$. We may form a three-manifold $S^{3}$ with embedded links by giving two solid tori as before, and then the resulting element $V(M) \in \mathbb{C}$ is given by

$$
\left\langle s_{(\varrho+\lambda) / k}^{-}, S_{*} s_{\left(\varrho+\lambda^{\prime}\right) / k}^{-}\right\rangle=\left(S_{*}\right)_{(\varrho+\lambda) / k,\left(\varrho+\lambda^{\prime}\right) / k} .
$$

In particular, for $G=S U(2)$ we reproduce the entire table (4.39) in [12]:

$$
V\left(S^{3}, L_{p} \cup L_{q}\right)=\frac{1}{\sqrt{k}} \sin \frac{\pi p q}{k} .
$$

for the invariants associated to $S^{3}$ with two unknotted, linked circles labeled by integers $p, q \leqq k$. 


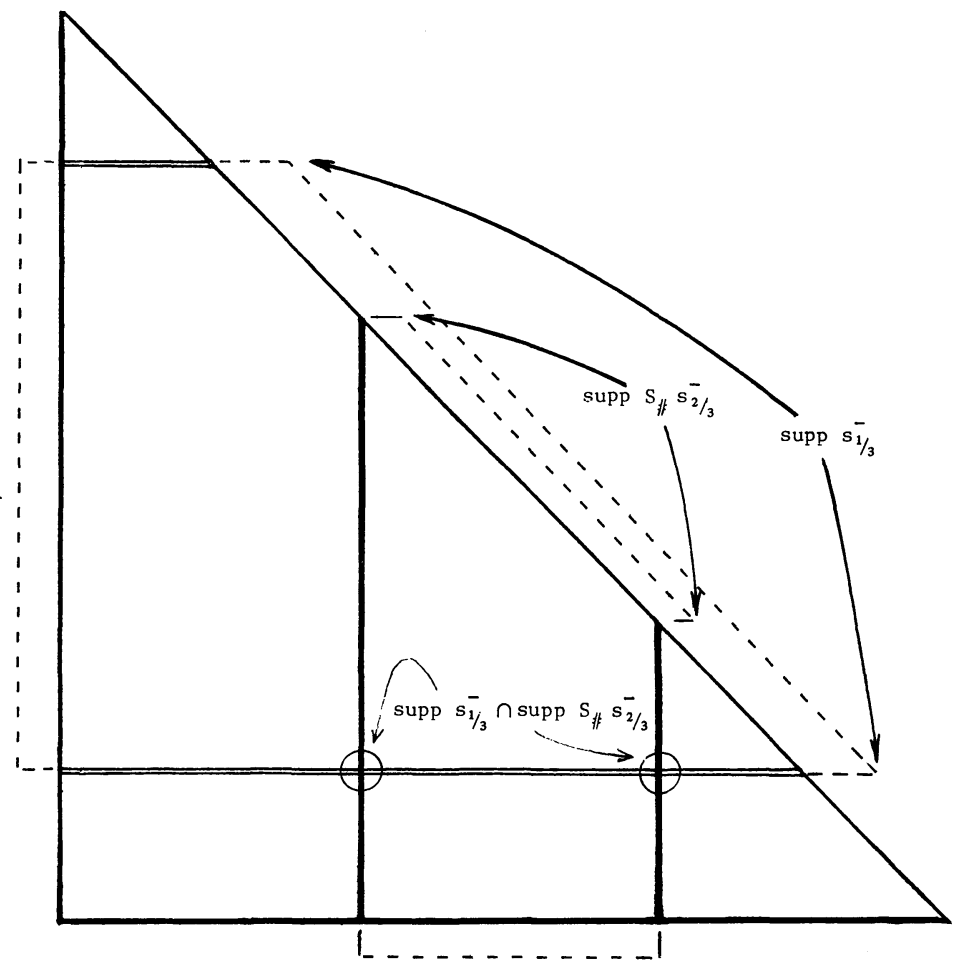

Fig. 2. The moduli space $\bar{S}_{1}$ for $G=S U(2)$, with the support of the sections $s_{1 / 3}^{-}$and $S_{\#}^{-} s_{2 / 3}^{-}$. The invariant $V\left(S^{3}, L\right)$, where $L$ is an unknotted circle, may be computed as the sum of the inner products of the values of the two sections at the points where their supports meet

The computation of $V(M, L)$ for $M$ given by a Heegaard splitting along a torus gives rise to an interesting interpretation of these objects. The sections $S_{(\varrho+\lambda) / k}^{-}$and $S_{\#} S_{\left(\varrho+\lambda^{\prime}\right) / k}^{-}$are supported on transverse Lagrangian subvarieties of $\bar{S}_{1}$. We take their inner product by formula (4.3), so that the invariant $V(M, L)$ is obtained by taking the inner product (in $\mathscr{L}$ ) of the values of the sections $\left.s_{(\varrho}^{-}+\lambda\right) / k$ and $S_{\#} s_{\left(\varrho+\lambda^{\prime}\right) / k}^{-}$ at each point where their supports intersect, and summing over intersection points (see Fig. 2). Thus the invariant is the sum of quantities computed at a discrete set of connections in $\tilde{S}_{1}$. In the case of $M=S^{3}$ and $G=S U(2)$ there are precisely two connections which contribute to this sum. In this way the invariant would seem to be analogous to the invariants of Casson and Johnson. However, the connections in question, rather than extending flatly to $M$, extend to $M$ as connections with singular curvature along two linked, unknotted circles. This behaviour and the analogy with the Casson invariants are obscured in the holomorphic formulation. However, while the set of flat connections on a three manifold $M$, which appears in the computation of the Casson invariant, can certainly be characterized without resorting to a Heegaard splitting, I do not know of any way of producing a similar invariant characterization of the connections appearing in the expression for $V(M)$. It is therefore not at all clear from this formulation that the $V(M)$ are independent of the Heegaard splitting.

We can however use our construction to produce true invariants of knots in $S^{3}$. For convenience we restrict our attention to the groups $S U(n)$, though this is by no means essential. We label the knot by an integral element $\lambda$ of the root lattice of 
$S U(n)$, corresponding to a choice of a representation. Let $K \subset S^{3}$ be a knot, and let $X \subset S^{3}$ be a neighbourhood of $K$ diffeomorphic to $S^{1} \times D^{2}$. Let $Y=S^{3} \backslash X$; we will write $S^{3}=X \cup_{\Sigma} Y$, noting that when $K$ is a nontrivial knot this will be a Heegaard splitting. Let $\Psi_{Y} \subset S L(2, \mathbb{Z})$ be the set of elements of $S L(2, \mathbb{Z})$ carrying the Lagrangian vector subspace of $H^{1}\left(\Sigma^{1}=\partial Y, \mathbb{R}\right)$ corresponding to $L_{Y} \subset \bar{S}_{1}$ to the standard Lagrangian subvariety corresponding to $L_{S^{1} \times D^{2}}$. Any two elements of $\Psi_{Y}$ differ by multiplication by $T^{m}$ for some integer $m$. Given an integer $k$, we have defined in Sect. IV a representation of $S L(2, \mathbb{Z})$; so given a choice of element $\psi \in \Psi_{Y}$, we define

$$
P_{\psi}(k)=\left(\left(\psi_{Y} S\right)_{*}\right)_{\varrho / k,(\varrho+\lambda) / k} .
$$

A different choice of $\psi^{\prime} \in \Psi_{Y}$ will yield an invariant $P_{\psi^{\prime}}(k)$ differing from $P_{\psi}(k)$ by a factor of $q^{m}$, where $q=q(k)=\exp (i \pi / 2 k)$, and $m$ is an integer. To make a precise statement, we will use the following definition. Let $f: \mathbb{Z} \rightarrow \mathbb{C}$ be a function. The balance class of $f$ is defined as the set of functions $\hat{f}: \mathbb{Z} \rightarrow \mathbb{C}$ satisfying $\hat{f}(k)$ $=q(k)^{m} f(k)$ for some integer $m$. By the previous remarks we see then that the elements of $\Psi_{Y} \subset S L(2, \mathbb{Z})$ define functions lying in a single balance class; let us denote this class by $P_{\Psi_{Y}}$. We may summarize this construction in the following proposition.

Proposition 5.1. The balance class $P_{\Psi_{Y}}$ is an ambient isotopy invariant of the knot $K$.

We note that in this case the splitting of $S^{\mathbf{3}}$ into two three-manifolds with boundary is given directly by the knot, and that therefore the issue of independence of choice of such splitting does not arise.

It would be interesting to understand the relation between these invariants and standard knot polynomials. A first step would be to generalize the invariants defined above to links.

Acknowledgements. I would like to thank I. M. Singer and Victor Guillemin for their helpful advice on the material contained in this paper.

\section{References}

1. Atiyah, M.: Michaelmas notes, unpublished

2. Atiyah, M., Bott, R.: The Yang-Mills equations over a Riemann surface. Phil. Trans. R. Soc. A 308, 523 (1982)

3. Axelrod, S., della Pietra, S., Witten, E.: Geometric quantization of Chern-Simons Gauge theory. Preprint

4. Elitzur, S., Moore, G., Schwimmer, A., Seiberg, N.: Remarks on the canonical quantization of the Chern-Simons-Witten theory. Preprint

5. Johnson, D.A.: Geometric form of Casson's invariant and its relation to Reidemeister torsion, unpublished

6. Kac, V.: Infinite dimensional Lie algebras. Cambridge: Cambridge University Press 1985

7. Mumford, D.: Abelian varieties. Oxford: Oxford University Press 1970

8. Ramadas, T.R., Singer, I.M., Weitsman, J.: Some comments on Chern-Simons Gauge theory. Commun. Math. Phys. 126, 409 (1989)

9. Segal, G.: The definition of conformal field theory, unpublished

10. Singer, I.M., Weitsman, J.: Real polarization of the moduli space of flat connections on a Riemann surface. Preprint

11. Sniatycki, J.: Geometric quantization and quantum mechanics. Berlin, Heidelberg, New York: Springer 1987

12. Witten, E.: Quantum field theory and the Jones polynomial. Commun. Math. Phys. 121, 351 (1989)

13. Woodhouse, N.: Geometric quantization. Oxford: Oxford University Press 1980

Communicated by A. Jaffe 